\title{
Literacia para a saúde: habilidades para lidar com as informações sobre saúde podem ajudar a construir novos caminhos na saúde pública
}

\author{
Health Literacy: skills to deal with health information can help build new \\ paths in public health
}

Alfabetización en salud: las habilidades para lidiar com informaciones en
salud pueden ayudar a construir nuevos caminos en la salud pública



Conceito transdisciplinar novo no cenário acadêmico brasileiro, a literacia em saúde, ou literacia para a saúde (LS), vem atraindo crescente interesse de pesquisadores de diversas áreas. A partir da experiência como coordenadora do grupo de estudos e pesquisa Promoção em Comunicação, Educação e Literacia para a Saúde no Brasil (ProLiSaBr), vinculado à Universidade Federal do Triângulo Mineiro (UFTM), a professora e pesquisadora Rosane Aparecida de Sousa destaca a importância do tema para a promoção da saúde da população. Em entrevista à Reciis, ela esclarece que o desenvolvimento de habilidades para buscar, acessar, compreender e avaliar as informações sobre saúde, aplicando-as para melhorar a qualidade de vida, pode ser também um caminho para o exercício da cidadania. "À medida que a população tem maior nível de literacia para a saúde, ela também tem maior capacidade e interesse de participação social, ela se reconhece como sujeito de direito". Seja como recurso para combater a desinformação ou como ferramenta de trabalho para profissionais da saúde, a LS desponta como opção para a construção de novos caminhos na saúde pública brasileira, destaca. Rosane Aparecida de Sousa possui pós-doutorado em Serviço Social pela Escola Nacional de Saúde Pública da Universidade Nova de Lisboa, é colaboradora do Programa de Residência Integrada e Multiprofissional em Saúde (RIMS) da UFTM e atuou como editora associada da Reciis de setembro de 2017 a dezembro de 2018.

Palavras-chave: Letramento em saúde; Promoção da saúde; Educação em saúde; Comunicação em saúde; Competência em informação. 
A new transdisciplinary concept in the Brazilian academic scenario, Health Literacy has been attracting increasing interest from researchers from different areas. Based on her experience as coordinator of the study and research group Promotion in Communication, Education and Literacy for Health in Brazil (ProLiSaBr), linked to the Federal University of Triângulo Mineiro (UFTM), professor and researcher Rosane Aparecida de Sousa highlights the importance of this theme to the promotion of health in the population. In an interview given to Reciis, she clarifies that the development of skills to seek, access, understand and evaluate health information, applying it to improve the quality of life, can also be a way to exercise citizenship. "As the population reaches a higher level of Health Literacy, it also has higher capacity and interest in social participation, it recognizes itself as a subject of law". Whether as a resource to combat misinformation or as a work tool for health professionals, Health Literacy emerges as an option for building new paths in Brazilian public health, she highlights. Rosane Aparecida de Sousa has a post-doctoral degree in Social Work from the National School of Public Health at Universidade Nova de Lisboa, is a contributor to the UFTM's Integrated and Multiprofessional Residency Program in Health and acted as associate editor of Reciis from September 2017 to December 2018.

Keywords: Health Literacy; Health promotion; Health education; Health communication; Information literacy.

Un nuevo concepto transdisciplinario en el escenario académico brasileño, la alfabetización en salud viene atrayendo el interés creciente de investigadores de diferentes áreas. A partir de su experiencia como coordinadora del grupo de estudio e investigación Promoción en Comunicación, Educación y Alfabetización para la Salud en Brasil (ProLiSaBr), vinculado a la Universidad Federal del Triângulo Mineiro (UFTM), la profesora e investigadora Rosane Aparecida de Sousa destaca la importancia del tema para la promoción de la salud de la población. En entrevista a Reciis, aclara que el desarrollo de habilidades para buscar, acceder, comprender y evaluar información en salud, aplicándola para mejorar la calidad de vida, también puede ser una forma de ejercer la ciudadanía. "En la medida en que la población tiene mayor nivel de alfabetización en salud, también tiene mayor capacidad e interés en la participación social, se reconoce como sujeto de derecho". Sea como recurso para combatir la desinformación o como herramienta de trabajo para los profesionales de la salud, la AS surge como em opción para construir nuevos senderos en la salud pública brasileña, destaca la autora. Rosane Aparecida de Sousa tiene posdoctorado en Trabajo Social de la Escuela Nacional de Salud Pública de la Universidade Nova de Lisboa, es colaboradora del Programa de Residencia Integrada y Multiprofesional de la UFTM y actuó como editora asociada de Reciis de septiembre de 2017 a diciembre de 2018.

Palabras clave: Alfabetización en salud; Promoción de la salud; Educación en salud; Comunicación en salud; Alfabetización informacional. 


\section{Reciis: A sua trajetória acadêmica inclui graduação e pós-graduações na área de serviço} social. Como o tema da comunicação na área da saúde atravessou sua pesquisa?

Rosane Sousa: Meu primeiro emprego como assistente social foi na Fundação de Ensino e Pesquisa de Uberaba (FUNEPU), vinculada à Universidade Federal do Triângulo Mineiro (UFTM). Naquele momento, em 1993, fui trabalhar no contexto hospitalar e fiquei muito incomodada com o fato de que na alta complexidade não temos muito espaço para trabalhar a promoção da saúde. Ali, o indivíduo já chega doente, recebe o diagnóstico, e todo o atendimento está focado na doença. Aquilo era algo que me chamava atenção: os pacientes, já adoecidos, faziam o tratamento, e depois não se tinha informações sobre o que havia acontecido com aquelas pessoas. Não havia uma articulação com a rede ou com as ações na área de promoção da saúde.

Em 2012, durante um congresso, encontrei, em uma banca de livros, o livro Comunicação e saúde, das professoras Inesita Soares de Araújo e Janine Miranda Cardoso (ARAÚJO; CARDOSO, 2007). Depois de ler o livro e vários artigos, dissertações e teses, especialmente vinculadas ao Instituto de Comunicação e Informação Científica e Tecnológica em Saúde (Icict) da Fundação Oswaldo Cruz (Fiocruz), resolvi escrever um projeto para concorrer a um edital da Fundação de Amparo à Pesquisa do Estado de Minas Gerais (Fapemig) para projetos de extensão e pesquisa. O nome do projeto foi Comunicação, Educação e Mobilização Social na Área de Saúde: Conhecendo os Recursos e Efetivando Direitos. O projeto foi aprovado pela Fapemig e desenvolvido ao longo de 2013 e 2014.

Ao final da primeira etapa, em novembro de 2013, foi realizado um seminário com a participação da doutora Janine, que proferiu a conferência de abertura sobre a comunicação na área de saúde. Tal evento contribuiu para a escolha desta temática como objeto de estudo no meu projeto para o pós-doutorado em Portugal.

Reciis: Além da comunicação e saúde, sua produção acadêmica aborda a formação profissional em saúde, a educação popular em saúde e, nos últimos anos, teve como foco a literacia. O que podemos entender como literacia em saúde ou literacia para a saúde (LS)?

Rosane Sousa: Esse é um termo novo no Brasil. No entanto, a partir de 2014 é possível identificar a ampliação das publicações usando essa terminologia nos artigos científicos. A LS pode ser entendida como uma ferramenta para trabalhar junto à população em geral e aos profissionais. Consiste de estratégias para que as pessoas possam desenvolver habilidades para: ter acesso às informações fidedignas, em geral, e especialmente no âmbito da saúde; ter a capacidade de compreender essas informações, identificando - por exemplo, nesses tempos de pandemia - o que é verdadeiro e o que não é, em termos de dados e informações sobre saúde, doenças e tratamentos; fazer a gestão das informações, conforme a realidade de cada um e as suas demandas; e, a partir desse movimento de acesso, compreensão e gestão da informação, identificar formas de investir em ações com enfoque na promoção da saúde e na qualidade de vida. Então, a LS vem como algo que vai compor esse rol de estratégias no campo da saúde, para que os profissionais, especialmente da saúde, possam trabalhar com a população o desenvolvimento de habilidades e capacidades para o cuidado e o investimento em saúde. 
Reciis: Você coordena o grupo de estudos e pesquisa Promoção em Comunicação, Educação e Literacia para a Saúde no Brasil, o ProLiSaBr, criado em 2014 para fomentar as reflexões e a produção de conhecimento no âmbito da promoção da literacia e da saúde. Quais as principais linhas de pesquisa desse grupo hoje? A que ele se dedica?

Rosane Sousa: O ProLiSaBr é um grupo inscrito no Conselho Nacional de Desenvolvimento Científico e Tecnológico (CNPq) e tem uma organização e um regulamento acadêmico. O ProLiSaBr está vinculado ao processo de formação na graduação e na pós-graduação. O grupo se dedica à realização de projetos de pesquisa, atividades e projetos de extensão com ações em educação permanente em saúde, formação profissional, cursos de aperfeiçoamento e workshops para discentes e profissionais. Temos três linhas bem amplas para que possamos fazer a articulação dessa temática: educação, comunicação, tecnologias e literacia; interface educação, saúde, políticas públicas e literacia para a saúde; e políticas e práticas de educação, saúde e serviço social.

Reciis: O ProLiSaBr integra a Rede de Promoção da Literacia para a Saúde na Lusofonia (ProLiSa), e ocorrem estreitas colaborações com as pesquisas desenvolvidas pelo professor Luis Saboga-Nunes, do Instituto de Sociologia da Universidade de Educação em Freiburg, na Alemanha. Qual a importância dessa colaboração entre Brasil e outros países nos estudos sobre LS?

Rosane Sousa: A importância é total. Eu tive os primeiros contatos com essa temática durante o pós-doutorado, em 2014, no qual fui supervisionada pelo professor Saboga-Nunes. Quando buscamos hoje textos em português sobre LS, encontramos grande parte da produção oriunda de Portugal. Eles fazem e têm investido muito em ações e pesquisas, e, inclusive, há uma política de Estado com enfoque na LS. Então essa articulação do ProLiSaBr como o projeto ProLiSa tem possibilitado nosso acesso às principais discussões sobre a LS - não só na Europa, como em outras regiões - para identificar aquilo que pode ser interessante para os nossos estudos e para a fundamentação das nossas discussões. A partir dessa relação com a ProLiSa desenvolvemos atividades e produzimos conhecimentos, inclusive com aproximação com a Universidade de Educação de Freiburg, que tem discutido a LS com enfoque na criança, no adolescente e na escola.

Reciis: As produções do grupo ProLiSaBr utilizam a expressão literacia para a saúde, mas existem alguns debates terminológicos em relação à tradução do termo Health Literacy para o português brasileiro. A palavra aportuguesada 'literacia' não consta entre os descritores de saúde da Biblioteca Virtual em Saúde (BVS), que utiliza a expressão letramento em saúde. Há uma explicação semântica para a escolha da expressão literacia para a saúde?

Rosane Sousa: Isso vem de um estudo que o professor Saboga-Nunes já estava realizando anteriormente e da compreensão do sentido dessas preposições que ligam os termos literacia e saúde. No inglês não há necessidade de preposição, então é Health Literacy. Mas, em português, esses termos precisam de preposição. Quando falo de literacia 'em’ saúde, é como se fosse algo externo àquele sujeito; algo que vem de fora, que eu trago pronto, e que essa pessoa deve assimilar como algo importante para si. Então, por isso, a compreensão de que o termo literacia 'em' saúde, para os nossos propósitos, não seria 
interessante. Um outro termo que também pode aparecer, mas em menor grau, é literacia 'da' saúde que traz a ideia de algo que vem unicamente a partir do sujeito, interno a esse sujeito. Nessa perspectiva, estaríamos desconsiderando os determinantes e as determinações sociais em saúde. Então entendemos que também não atenderia ao propósito dos nossos estudos. A literacia 'para a' saúde seria a junção da literacia 'em' saúde e da literacia 'da' saúde (SABOGA-NUNES et al., 2019). A LS é a capacidade de um indivíduo ter acesso a informações, compreendê-las, geri-las a partir do contexto social onde está inserido, e a partir dali pensar formas de utilizar todo esse arcabouço para investir na promoção da sua saúde. Essa concepção ratifica a autonomia do sujeito, de modo que ele possa estabelecer escolhas e construir perspectivas no âmbito da promoção da saúde, da qualidade de vida e do estilo de vida saudáveis, sem perder de vista os determinantes sociais em saúde.

Reciis: Com relação à tradução do termo literacy, existe alguma preferência, no português brasileiro, por literacia, em vez de letramento ou alfabetização?

Rosane Sousa: Estes dois termos são extremamente importantes para que possamos pensar a promoção da saúde e trabalhar nessa perspectiva. Quando o grupo ProLiSaBr iniciou seus estudos e fizemos um levantamento da realidade do Brasil, em 2014, tínhamos uma produção bem menor de estudos sobre LS, e nos deparamos com o termo 'letramento em saúde'. O que identificamos nos estudos e artigos publicados é que a ideia de letramento em saúde está muito vinculada a uma perspectiva funcional: seria a preparação do indivíduo para atender a questões pontuais e necessárias para sua vida cotidiana, sem que seja exigido dele pensar criticamente ou mesmo se posicionar frente àquelas questões. Por exemplo: uma ação com enfoque no letramento funcional em saúde pode ser um profissional da saúde criar, nas embalagens de remédio, as indicações com desenhos para que um paciente que não saiba ler possa tomar a medicação corretamente - colocar uma ilustração de sol nascendo para o medicamento da manhã, uma lua para o da noite. Estou falando isso de uma forma muito reducionista, mas essas são formas de trabalhar com a população para atender a questões pontuais. A opção pelo termo literacia para a saúde nos remete ao desenvolvimento de capacidades desse indivíduo para que ele tenha autonomia de escolha e para que possa, a partir disso, ser sujeito da sua própria história. Com isso, espera-se que esse indivíduo, além de conseguir tomar seus medicamentos, conforme orientação médica, tenha espaço para acessar informações que possibilitem refletir sobre sua doença, seu tratamento e suas formas de promover a sua saúde e a daqueles com os quais convive.

Reciis: Nos seus textos, você afirma que a assistência em saúde se caracteriza, em grande medida, pelo modelo biomédico, centrado na doença. A perspectiva da LS, por outro lado, se alinharia com o paradigma salutogênico (SABOGA-NUNES et al., 2016), com foco na saúde. O que seria isso?

Rosane Sousa: Na realidade brasileira, ainda prevalece a ênfase na doença e no tratamento, com exames e medicamentos, reforçando uma perspectiva patogênica na área de saúde. Se olharmos para os currículos das profissões da área de saúde, como a medicina, por exemplo, um aluno tem carga horária muito menor sobre promoção da saúde; a maior parte da sua formação será sobre as doenças e os seus impactos, os sintomas e as formas de tratamento. Quando começamos a perceber isso, notamos que as ações e as intervenções na área de saúde - por exemplo, na atenção primária - ainda repercutem esse aspecto, pois estão centradas nos agentes causais da doença, em como eles atuam no organismo, têm por 
foco principal a análise das informações e o impacto do processo de adoecimento do sujeito. Isso é reforçado pelo fato de que o modelo biomédico que nós temos ainda enfatiza a figura do médico como aquele que vai determinar o que será feito mediante o diagnóstico do usuário ou do paciente. Os demais profissionais de saúde vêm como coadjuvantes no processo saúde-doença.

A salutogênese é uma teoria criada por Aaron Antonovsky (1990), israelense-americano que fez uma pesquisa com mulheres que viveram o período do Holocausto. Ele queria entender por que algumas mulheres tiveram resiliência frente à questão complexa e difícil do Holocausto, enquanto outras não suportaram e entraram em processo de depressão e adoecimento. A teoria da salutogênese parte de um conceito ampliado de saúde, busca a superação da dicotomia saúde/doença e enfatiza o desenvolvimento das competências individuais e coletivas para que cada um de nós possa aumentar o próprio controle sobre a vida e a saúde. Antonovsky aponta que o indivíduo tem recursos pessoais protetivos, formas de reagir para a autoproteção. Esses recursos é que nos permitem tomar decisões diante de elementos internos ou externos a nós. Assim, se partimos do princípio da saúde, se penso em uma vida organizada, a partir das condições de saúde necessárias para que se viva bem e com qualidade de vida, vou trabalhar para que as pessoas invistam cada vez mais em ações e atitudes que possam contribuir para que essa saúde permaneça. São ações do cotidiano, por exemplo: alimentação saudável, atividades físicas, beber água. Esses aspectos vão auxiliar para que eu, já saudável, possa manter, cada vez mais, melhores condições de saúde e de qualidade de vida. A perspectiva salutogênica, com enfoque na saúde, nos possibilita trabalhar com as pessoas e reconhecer nelas os potenciais para que elas possam investir na própria saúde e se fortalecer, mesmo considerando os impactos da realidade social e do contexto social complexo e difícil que estamos vivendo.

\section{Reciis: Em 2019, você organizou e lançou, com outros três pesquisadores, o livro O papel da literacia para a saúde e educação para a saúde na promoção da saúde (SABOGA- NUNES et al., 2019). A LS pode ser considerada uma estratégia e também um resultado da promoção da saúde?}

Rosane Sousa: Eu creio que a LS seja uma estratégia, quando pensamos que pode ser um caminho para que a população se reconheça como sujeito de diretos. A partir do acesso e da compreensão da informação, da participação e da mobilização social, acredito que esses cidadãos possam reivindicar, então, que o Estado assuma a sua responsabilidade no financiamento e no desenvolvimento de ações e projetos com enfoque não apenas na saúde, mas nas diversas áreas, atendendo àquilo que está previsto na Constituição Federal. A partir do movimento dos cidadãos e do Estado, a LS pode também ser considerada um resultado. Por quê? Pode ser resultado, no âmbito da promoção da saúde, a partir do desenvolvimento de habilidades e competências de cada um e do coletivo no cuidado da saúde individual e no cuidado da comunidade e, ao mesmo tempo, ter consciência e clareza para fazer a leitura crítica da realidade social em que vivemos e cobrar do Estado o cumprimento das suas responsabilidades no âmbito das políticas públicas. Esse movimento pode ser articulado. A LS pode ser a estratégia necessária para avançarmos no âmbito da promoção da saúde, e, quando conseguirmos - porque hoje ainda não temos essa realidade -, quando dermos outros passos para fomentar essa participação e mobilização social - ou seja, esse acesso à informação e ao conhecimento -, teremos como consequência uma melhora nas ações de promoção da saúde e na forma com que os usuários vão agir, no sentido de cuidar da sua saúde. 
Reciis: A partir dos anos 2000, a LS passou a ser vista pela Organização Mundial da Saúde (OMS) como um importante objetivo da saúde pública e um componente fundamental em busca da saúde e do bem-estar na sociedade moderna (KICKBUSCH et al., 2013). A efetivação do direito à saúde da população, a partir da autonomia e da participação social, passa necessariamente por altos níveis de LS?

Rosane Sousa: Penso que, assim como na questão anterior, podemos ter aqui uma via de mão dupla. Se considerarmos a realidade social brasileira hoje, na qual prevalece a iniquidade social, a desigualdade na distribuição de renda e a fragilização do acesso aos direitos sociais (especialmente na área de saúde), precisamos de um trabalho junto à população, por meio da educação popular em saúde, de modo a possibilitar o acesso a informações - que hoje é urgente. Refiro-me ao acesso às informações e aos conhecimentos fidedignos, que esclareçam, que deem suporte para que as pessoas entendam o que de fato está acontecendo no âmbito da saúde de um modo geral, e à criação de espaços de participação social, reflexões, para que a população como um todo se reconheça como sujeito de direitos. Aliado a isso, à medida que trabalhamos nessa perspectiva, por meio da mobilização e da participação dos cidadãos, temos mais condições de reivindicar que o Estado assuma sua responsabilidade com a efetivação das políticas públicas, bem como os direitos e as garantias sociais. Esses dois contextos podem contribuir para a ampliação dos níveis de LS. À medida que cresce o nível de LS da população, ela passa a ter também mais autonomia, mais capacidade e interesse de participação social.

Reciis: Apesar de ser um campo de investigação relativamente novo, já existem instrumentos de medição da LS traduzidos para o português brasileiro e validados no Brasil. Fale um pouco sobre o que vocês utilizam no ProLiSaBr: o quanto esses instrumentos nos dizem sobre as habilidades das pessoas em lidar com as informações sobre saúde? Quais as potencialidades e as limitações dos questionários?

Rosane Sousa: No grupo ProLiSaBr, até por essa aproximação com o ProLiSa, tivemos o primeiro contato com o instrumento HLS-EU (European Health Literacy Survey) (SØRENSEN et al., 2013) e, depois, com o HLS-EU-PT (SABOGA-NUNES et al., 2014), que foi uma tradução do inglês. Esse instrumento foi elaborado por um consórcio europeu e aplicado em alguns países do continente; depois, o professor Saboga-Nunes fez a tradução e a transculturação para o português de Portugal. Quando eu estava no pós-doutorado, trabalhei com ele no processo de transculturação para o português brasileiro (HLS-EUBR) (MARTINS; SABOGA-NUNES, 2014). Esse instrumento específico tem 47 questões e nos possibilita fazer uma análise do nível de literacia geral e, depois, nos oferece condições de identificar, por meio das respostas, o nível de literacia dos participantes quanto aos cuidados em saúde, na prevenção de doenças e na promoção da saúde. Portanto, temos a possibilidade de identificar quatro níveis de LS, e dentro de cada um deles podemos identificar se aquele grupo pesquisado tem um nível de literacia insuficiente, problemático, regular ou excelente. Como toda pesquisa quantitativa, esse instrumento conta com parâmetros sobre como identificar essas questões. Esses aspectos são interessantes porque podem nos mostrar, por exemplo, que determinado grupo tem um bom nível de literacia em prevenção de doenças e cuidados em saúde, mas nível insuficiente em promoção da saúde - o que é o mais comum.

Nas pesquisas que já realizamos no grupo ProLiSaBr temos um número significativo de respostas com essa característica. A limitação desses questionários, como em toda pesquisa quantitativa, é que eles generalizam. Então, você aplica um questionário e não tem condição de identificar outros determinantes 
que possam estar implicando nas condições de saúde ou mesmo nos níveis de LS daquela população. Cada vez que temos um resultado, precisamos relacionar as informações e contextualizá-las com a realidade social que estamos nos propondo a pesquisar.

Reciis: No livro Literacia em saúde, lançado em 2021 pela Editora Fiocruz (PERES; RODRIGUES; SILVA, 2021), os autores afirmam que a LS deveria ser mais desenvolvida e menos mensurada. Você concorda?

Rosane Sousa: Em relação à realidade brasileira, não temos ainda uma política pública de saúde com enfoque nas ações de LS. Diante disso, creio que as pesquisas e intervenções podem ocorrer à medida que passarmos a ter um panorama do nível de LS no Brasil. É diferente, por exemplo, de Portugal, onde o Estado assumiu a LS como política e vem liberando editais para que grupos de pesquisadores possam realizar suas pesquisas no país. Lá eles já têm um panorama do nível de LS da população. No Brasil ainda não temos isso, aqui as pesquisas são pontuais, restritas a aspectos locais, regionais. Muitos dados estão relacionados ao letramento em saúde de pessoas com alguma comorbidade ou com doenças crônicas não transmissíveis, entre outras. Mas eu concordo que está na hora de avançarmos na proposição de ações, projetos, intervenções e de uma política pública para trabalhar com a população a ampliação de seus níveis de LS.

Reciis: No contexto da pandemia de covid-19, com a excessiva circulação de conteúdos sobre saúde vindos de diversas fontes, temas como infodemia e desinformação ganharam destaque. Como você avalia as contribuições dos estudos sobre LS em períodos pandêmicos, especialmente em relação às informações sobre saúde que circulam na internet e nas mídias digitais?

Rosane Sousa: Avalio como algo ainda muito introdutório. Como mencionei, estamos realizando pesquisas muito limitadas a respostas a situações pontuais. Quando começamos a vivenciar essa pandemia, no início de 2020, nós não tínhamos estrutura nem política pública, ou mesmo espaços para trabalhar com a população o acesso às informações e a compreensão das informações fidedignas necessárias para a construção de uma outra realidade diante da desinformação. Infelizmente, acredito que as contribuições dos estudos sobre LS no Brasil, durante a pandemia, não podem ser avaliadas porque não tínhamos ainda uma base sólida que pudesse dar um suporte para as diversas instituições ou mesmo para as diversas mídias digitais que trabalhassem nesse sentido. Por exemplo: no grupo ProLiSaBr realizamos, em julho de 2020, um workshop on-line voltado para profissionais e alunos que teve grande demanda. Nos primeiros encontros, percebemos o quanto as pessoas estavam impactadas com a pandemia. Reconheço que seria extremamente importante que tivéssemos mais espaços, estrutura e recursos humanos para trabalhar não só naquele momento - que foi muito importante -, mas mesmo hoje, em que ainda assistimos a uma ampliação da desinformação sobre questões extremamente importantes, não só na área de saúde, mas no âmbito das políticas públicas e dos direitos sociais. 
Reciis: Para onde devem apontar as próximas investigações sobre LS realizadas no Brasil? Existe, talvez, uma questão principal, ainda não respondida, em relação à literacia e aos seus atravessamentos com a saúde?

Rosane Sousa: Em junho de 2021, o grupo ProLiSaBr participou de uma chamada de projetos, e, então, tivemos conhecimento de que o Ministério da Saúde, em parceria com a Organização Pan-Americana da Saúde (Opas), tinha interesse em aprofundar as ações sobre LS no Brasil. A partir de uma articulação do grupo ProLiSaBr com profissionais da Fiocruz, apresentamos uma proposta para desenvolver um curso autoinstrucional para os profissionais da área de saúde, com enfoque na LS. Nossa proposta foi aprovada e vamos realizar, em 2022, um projeto sobre autocuidado em saúde, qualidade de vida e estilo de vida saudável para trabalhadores da saúde vinculados à Atenção Primária à Saúde (APS), no qual faremos a formação de profissionais para pensar a LS como uma ferramenta que pode ser utilizada na APS. Acredito que esse possa ser o ponto de partida para que a LS se torne uma política pública, caso os profissionais da saúde reconheçam essa temática como importante. E aí, sim, teríamos uma ampliação de investigações sobre LS mais voltadas para o contexto da promoção da saúde, e não para o tratamento de doenças, como tem feito uma parte significativa das publicações no Brasil. Com isso, acredito que a gente possa avançar, não só em termos de investigações, mas na educação permanente em saúde para que os profissionais que estão na ponta tenham na LS mais uma ferramenta que possam utilizar no seu dia a dia.

Reciis: É relativamente comum a ausência de temas sobre comunicação e saúde e LS nos currículos dos cursos de área da saúde, tanto a nível de graduação quanto na pósgraduação. O quão importante seria incorporar esses temas na formação dos profissionais da saúde?

Rosane Sousa: Temos percebido a necessidade da sensibilização dos profissionais de saúde que estão nas coordenações e nos colegiados de cursos e nos Núcleos Docentes Estruturantes (NDEs) que discutem a revisão de projetos pedagógicos para a ideia de que nós precisamos avançar e preparar os profissionais para a promoção da saúde, e não só para o tratamento ou a prevenção de doenças. Se nós fizermos o caminho inverso, se chegarmos nos espaços de prática, como os estágios, e tivermos profissionais que comecem a atuar na promoção da saúde na perspectiva da salutogênese, da comunicação em saúde e da LS, poderemos despertar nos alunos a importância da promoção da saúde e das ações de fortalecimento da autonomia dos usuários e dos serviços de saúde - o que terá um impacto muito importante no cotidiano de trabalho, bem como no processo de formação na graduação e na pós-graduação. Mas, para isso, precisamos ainda romper com questões históricas que foram construídas sobre as profissões da área de saúde, ainda centradas no modelo biomédico, conforme apontou Koifman (2001). A possibilidade seria construir um novo caminho: investir mais na promoção da saúde. Mas ainda há um grupo importante de profissionais que resistem a mudanças mais amplas e profundas nos projetos pedagógicos dos cursos da área de saúde. Essa atitude, em alguns casos, é também fortalecida pelos conselhos vinculados a essas profissões.

Ao refletir sobre o processo de formação na área de saúde, as mudanças e os avanços com enfoque na promoção da saúde, comunicação em saúde, salutogênese e LS ainda são pontuais e algumas vezes incipientes. Novamente, a ideia do modelo biomédico centrado na patogenia, na doença, prevalece. À medida que vamos chegando nos diversos pontos (nos espaços de estágio e formação, como as residências multiprofissionais em saúde), levando esse conteúdo, criamos espaços com aqueles que comungam da mesma perspectiva, que acreditam no SUS, que querem trabalhar com a promoção da saúde. E vamos 
desenvolvendo nossas ações. É algo às vezes pequeno nas diversas universidades, mas é assim que rompemos algo que está cristalizado.

Reciis: A Fiocruz lançou recentemente o projeto Reconquista das Altas Coberturas Vacinais, que busca estabelecer uma rede entre vários atores para melhorar a cobertura vacinal brasileira. Em um artigo publicado em 2020 (MOREIRA; MARTINS, 2020), você afirma que a mensuração e o desenvolvimento da LS podem ser relevantes para reduzir o percentual de não vacinação. Como a LS poderia contribuir para o controle das doenças?

Rosane Sousa: A literacia para a saúde e a literacia para a saúde digital podem ser instrumentos valiosos para alcançar a população de uma forma mais ampla, ao esclarecer questões importantes relacionadas à vacinação, à sua cobertura e relacionadas também à importância de se garantir uma melhor condição de saúde e de qualidade de vida para todos. Esse artigo foi escrito por mim e pela mestra Kéllen Campos Castro Moreira, enfermeira que trabalha no setor de vacinas da Secretaria Municipal de Saúde de Uberaba (MG). O que percebemos é que, às vezes, a desinformação faz com que pessoas não vacinem seus filhos. Mas, quando trabalhamos o acesso à informação, a compreensão e a apropriação disso na vida de cada pessoa, as posturas começam a mudar. Precisamos, sobretudo, considerar uma ação mais ampla nas unidades de saúde, de forma que a gente possa dar acesso a informações, ouvir as pessoas e esclarecer as suas dúvidas, considerando a realidade sociocultural e o contexto no qual elas vivem. A partir do que estávamos estudando naquele momento, percebemos que a cobertura vacinal sofre uma redução na medida em que há um grupo populacional que não detém maiores informações e conhecimentos sobre aquele processo. Por isso, entendemos que a LS e a literacia para a saúde digital podem ser ferramentas importantes para, como diz Milton Nascimento, "ir até onde o povo está".

Reciis: A LS é um campo do conhecimento essencialmente transdisciplinar: estabelece diálogos teóricos entre áreas mais tradicionais, como a educação, a comunicação, as ciências da informação e a saúde. O quanto essa transdisciplinaridade favorece ou dificulta a evolução acadêmica das produções intelectuais sobre LS?

Rosane Sousa: Particularmente, pela experiência que tenho tido de 2014 para cá, no grupo ProLiSaBr, ou mesmo na relação com outros profissionais que entram em contato por conta do instrumento HLS-EU$\mathrm{BR}$, penso que a transdisciplinaridade só tem aspectos positivos. A experiência que temos hoje no ProLiSaBr é muito rica - temos odontólogo, sociólogo, antropólogo, assistente social, economista, enfermeira, alunos de graduação e pós-graduação em diversas áreas. Quando vamos discutir a concepção de saúde, por exemplo, para pensarmos o desenvolvimento de um projeto de extensão ou de um workshop, temos visões diversas e aprendemos uns com os outros. Os que estão no grupo vão para as suas áreas e discutem essas temáticas, o que também vai despertando outras pessoas. Vejo que essa categoria teórica, a literacia para a saúde, pode possibilitar um trabalho transdisciplinar que favoreça a discussão e a implementação de ações nas diversas políticas públicas, e não só na área de saúde. Por exemplo, uma área na qual ultimamente desenvolvemos pesquisas é a área de educação do Ensino Médio. Temos uma pessoa no grupo que está fazendo doutorado na Argentina e realiza pesquisa com professores. Isso possibilita discutir e debater esses aspectos de forma mais ampla, mais democrática e muito mais rica, para que possamos avançar em termos de produção de conhecimento e de identificação da nossa realidade quanto aos níveis de LS da população brasileira. 
Reciis: Acerca da atual literatura sobre a LS: podemos dizer que já é suficientemente sólida e robusta, ou ainda incipiente? Especificamente no Brasil, existem autores brasileiros que já podem ser considerados referência nesse debate?

Rosane Sousa: Acho que em termos internacionais, sim. Temos uma gama de publicações em periódicos respeitadíssimos no contexto acadêmico. Temos experiência de desenvolvimento de ações e de políticas públicas em alguns países que nos mostram o quanto é possível e viável construir uma proposta de atuação em nível nacional. Em termos de Brasil, não temos ainda uma produção robusta ou mesmo autores de referência, quando se fala em LS. Primeiro porque temos vários grupos sedimentados que vieram trabalhando sobre letramento em saúde - grupos que foram e são muito importantes. Observa se, por meio das publicações, o uso dos termos 'letramento em saúde' e 'literacia para a saúde' como sinônimos. À medida que ocorra a apropriação sobre as discussões acerca da LS é possível identificar as diferenças ideopolíticas que circundam o uso do termo. A discussão filológica do conceito de Health Literacy, das várias correntes e escolas internacionais que realizam pesquisas e publicações com este recorte e a identificação dos autores de referência para esta categoria teórica possibilitam a compreensão da concepção da literacia para a saúde.

Outro aspecto é que, no Brasil, há um número reduzido de pesquisadores investigando essa temática. Hoje temos como referência a professora Maria Rosilene Cândido Moreira, da Universidade Federal do Cariri (UFCA), que tem participado conosco, trabalha na pós-graduação e tem se aprofundado nessas discussões. Temos um grupo na Universidade Regional do Noroeste do Estado do Rio Grande do Sul (UniJuí) composto por Graça Simões de Carvalho, Eva Teresinha de Oliveira Boff e Maria Cristina Pansera-de-Araújo. Esse grupo tem aproximação com o professor Luis Saboga-Nunes. Na Fiocruz, destacam-se a médica pesquisadora Ana Luiza Braz Pavão (PAVÃO et al., 2021) e o professor Frederico Peres (PERES; RODRIGUES; SILVA, 2021). Vejo também algumas publicações na Universidade Federal de Santa Catarina (UFSC) e na Universidade Federal do Rio Grande do Sul (UFRGS). Destaca-se o livro que publicamos: O papel da literacia para a saúde e educação para a saúde na promoção da saúde (SABOGA-NUNES et al., 2019), além de artigos e dissertações abordando a literacia digital para a saúde (SOUSA et al., 2020; LEÃO, 2020).

Ainda temos muito caminho pela frente e espaços para que possamos investir em pesquisa e produção de conhecimentos sobre a LS. Desta forma, a médio prazo, teremos publicações genuinamente brasileiras sobre essa temática.

\section{Reciis: Você diria que a LS é um tema que atrai crescente interesse no atual contexto acadêmico brasileiro?}

Rosane Sousa: Creio que sim. Eu me lembro que em 2014, quando fui fazer buscas sobre LS em textos publicados sobre o tema no Brasil, encontrei pouquíssimos artigos. Hoje, cadastrei o termo no Google Acadêmico e em outras ferramentas, e toda semana recebo notificação de alguma nova publicação - seja em congressos, dissertações, TCCs, artigos publicados. Então, vejo que estamos num movimento crescente no Brasil na abordagem da categoria teórica literacia para a saúde (PAVÃO; WERNECK, 2021). A atual realidade social tem mostrado aos profissionais da saúde, especialmente, a importância e a necessidade de rompermos com essa situação na qual os usuários da saúde são colocados numa condição de subserviência. É preciso que a população tenha um papel ativo como sujeito social. E isso vai ser importante não apenas para esse cidadão, mas para os profissionais de saúde, para os serviços de saúde, 
para o nível de promoção da saúde dessa população. Creio, então, que possamos despertar interesse nas pessoas para essa temática, para que possamos, juntos, construir possibilidades de ação, intervenção e pesquisa a partir da ampliação dos níveis de LS da população brasileira.

\section{REFERÊNCIAS}

ANTONOVSKY, Aaron. The salutogenic model of health. New York: Guilford Press, 1990.

ARAÚJO, Inesita Soares de; CARDOSO, Janine Miranda. Comunicação e saúde. Rio de Janeiro: Editora Fiocruz, 2007.

KICKBUSCH, llona et al. (ed). Health Literacy: the solid facts. Copenhague: World Health Organization, 2013. Disponível em: https://apps.who.int/iris/handle/10665/326432. Acesso em: 10 fev. 2022.

KOIFMAN, Lilian. O modelo biomédico e a reformulação do currículo médico da Universidade Federal Fluminense. História, Ciências, Saúde - Manguinhos, Rio de Janeiro, v. 8, n. 1, p. 48-70, 2001. DOI: https://doi.org/10.1590/S0104-59702001000200003. Disponível em: https://www.scielo.br/j/hcsm/al wbJxmgpRcpNXYjChnxzVWps/abstract/?lang=pt. Acesso em: 18 fev. 2022.

LEÃO, Nicole Fajardo. eHealth Literacy entre jovens: estudo exploratório sobre o papel das condições socioeconômicas no uso da informação sobre saúde na internet. 2020. 196 f. Dissertação (Mestrado em Informação e Comunicação em Saúde) - Instituto de Comunicação e Informação Científica e Tecnológica em Saúde, Fundação Oswaldo Cruz, Rio de Janeiro, 2020. Disponível em: https://www.arca.fiocruz.br/handle/ icict/44437. Acesso em: 10 fev. 2022.

MARTINS, Rosane Aparecida de Sousa; SABOGA-NUNES, Luis. The challenges of epistemological validation to Brazil of the European Health Literacy Survey (HLS-EU-BR). Atención Primaria, Barcelona, v. 46, n. espec. cong. 1, p. 12, 2014. Trabalho apresentado no $2^{\text {nd }}$ World Congress of Health Research, 2014, Viseu. Disponível em: https://www.elsevier.es/es-revista-atencion-primaria-27-pdf-S0212656714700691. Acesso em: 10 fev. 2022.

MOREIRA, Kéllen Campos Castro; MARTINS, Rosane Aparecida de Sousa. A não vacinação dos filhos e a Literacia para a Saúde. REFACS, Uberaba (MG), v. 8, supl. 3, p. 1055-1063, 2020. DOI: https://doi. org/10.18554/refacs.v8i0.5022. Disponível em: https://seer.uftm.edu.br/revistaeletronica/index.php/refacs/ article/view/5022. Acesso em: 10 fev. 2022.

PAVÃO, Ana Luiza Braz et al. Avaliação da literacia para a saúde de pacientes portadores de diabetes acompanhados em um ambulatório público. Cadernos de Saúde Pública, Rio de Janeiro, v. 37, n. 10, p. e00084819, 2021. DOI: https://doi.org/10.1590/0102-311X00084819. Disponível em: https://www.scielo.br/j/ csp/a/tMLFp5Wk9StnrhMg4tB33sg/?lang=pł\#. Acesso em: 10 fev. 2022.

PAVÃO, Ana Luiza Braz; WERNECK, Guilherme Loureiro. Literacia para a Saúde em países de renda baixa ou média: uma revisão sistemática. Ciência \& Saúde Coletiva, Rio de Janeiro, v. 26, n. 9, set. 2021. DOI: https:// doi.org/10.1590/1413-81232021269.05782020. Disponível em: https://www.scielo.br/j/csc/a/Mv5VvPM58ryKM wzX6KDBypQ/?lang=pt. Acesso em: 10 fev. 2022.

PERES, Frederico; RODRIGUES, Karla Meneses; SILVA, Thais Lacerda e. Literacia em saúde. Rio de Janeiro: Editora Fiocruz, 2021.

SABOGA-NUNES, Luis et al. Cultural adaptation and validation to Portuguese of the European Health Literacy Survey (HLS-EU-PT). Atención Primaria, Barcelona, v. 46, n. espec. cong. 1, p. 13, 2014. Trabalho apresentado no $2^{\text {nd }}$ World Congress of Health Research, 2014, Viseu. Disponível em: https://www.elsevier.es/ es-revista-atencion-primaria-27-pdf-S0212656714700691. Acesso em: 10 fev. 2022.

SABOGA-NUNES, Luis et al. Níveis de alfabetização/Literacia para a Saúde em duas populações de diferentes níveis de escolaridade na construção da cidadania. In: BOFF, Eva Terezinha de Oliveira; PANSERADE-ARAÚJO, Maria Cristina; CARVALHO, Graça Simões de (org). Interações entre conhecimentos, valores e práticas na educação em saúde. ljuí: Editora Unijuí, 2016. p. 57-88. 
SABOGA-NUNES, Luis et al. (org). O papel da literacia para a saúde e educação para a saúde na promoção da saúde. Curitiba: Editora CRV, 2019. Disponível em: https://drive.google.com/file/ d/1 7jqG1QML69yG-cizK-plxN-eY9um6mv/view. Acesso em: 10 fev. 2022.

SØRENSEN, Kristine et al. Measuring Health Literacy in populations: illuminating the design and development process of the European Health Literacy Survey Questionnaire (HLS-EU-Q). BMC Public Health, Londres, v. 13, n. 948, out. 2013. DOI: https://doi.org/10.1186/1471-2458-13-948. Disponível em: https://bmcpublichealth.biomedcentral.com/articles/10.1186/1471-2458-13-948. Acesso em: 10 fev. 2022.

SOUSA, Rosane Aparecida de et al. Literacia digital e interfaces com a saúde: uma revisão integrativa. In: PEREIRA NETO, André; FLYNN, Matthew B. (org.). Internet e saúde no Brasil: desafios e tendências. São Paulo: Cultura Acadêmica, 2020. p. 422-444. 